Available online at www.eccomasproceedia.org

Eccomas Proceedia COMPDYN (2021) 2112-2120

\title{
VIRTUAL SENSING AND STRAIN ESTIMATION ON AN OFFSHORE WIND TURBINE USING SUPERVISED LEARNING
}

\author{
Marius Tarpø ${ }^{1}$, Sandro Amador ${ }^{1}$, Evangelos Katsanos ${ }^{1}$, Mattias Skog ${ }^{1}$, Johan Gjødvad ${ }^{2}$, \\ and Rune Brincker ${ }^{1}$ \\ ${ }^{1}$ Technical University of Denmark \\ Brovej B. 118, Kgs. Lyngby, Denmark \\ e-mail: mariotarpoe@gmail.com, sdio@byg.dtu.dk, vakat@byg.dtu.dk, runeb@byg.dtu.dk \\ ${ }^{2}$ Sigicom \\ Glasfibergatan 8, 12545 Älvsjö, Sweden \\ e-mail: mattias.skog@sigicom.com, johan.gjodvad@sigicom.com
}

\begin{abstract}
Virtual sensing enables expansion and transformation of measured quantities from physical sensors into new quantities at unmeasured locations. This allows for estimating the strain and stress in unmeasured locations of a system by transforming the physical sensors (input) into the desired strain and stress response (output). This transformation model can be based on either knowledge of the systems, data from the system, or any combination of these. In this paper, supervised learning and data-driven models are applied to strain estimation of an offshore wind turbine through Principal Component Analysis (PCA). Training data are used to establish the data-driven model that enables a versatile strain estimation that functions well under different wind scenarios than the training data set.
\end{abstract}

Keywords: Data-driven model, virtual sensing, stress estimation, structural health monitoring, principal component analysis 


\section{INTRODUCTION}

Virtual sensing transforms measured data into other quantities and/or expands the data to unmeasured locations through virtual sensors [1]. We can categorise stress/strain estimation technique as a subcategory within virtual sensing that estimates the full-field stress/strain response of a system [2]. The reader should note that there is unfortunately not a consensus or common terminology in the field of virtual sensing and stress/strain estimation [2].

Stress/strain estimation started in the 1950s with models of analytical relationships between response and strain [3]. In the 1990s, transformation matrices started to replace the analytical relationships with Okubo and Yamaguchi [4] introducing a displacement-to-strain transformation matrix, which is based on data of both strain and displacement. Hjelm et al. [5] used mode shapes to create a transformation matrix - called the modal expansion technique - that enabled full-field strain estimation. The techniques of stress/strain estimation were expanded to include adaptive filters as Papadimitriou et al. [6] introduced the Kalman filter to full-field strain estimation in 2011. In 2016, Maes et al. [7] compared the different techniques of the transformation matrix (modal expansion) and adaptive filters (Kalman filter and the joint inputstate estimation algorithm) for strain estimation on an offshore monopile wind turbine and they found the technique to be equal performance-wise. Accounting for the quasi-static response, Iliopoulos et al. [8] fused both accelerometers and strain gauges into the modal expansion technique while Skafte et al. [9] applied Ritz vectors to the modal expansion technique. Risaliti et al. [10] introduced the augmented extended Kalman filter on a nonlinear system using the implicit equation of motion. Tarpø et al. [11] studied expansions of experimental mode shapes used for stress estimation and they concluded that expansions of mode shapes is equivalent to updating the system model. Nabuco et al. [12] estimated the dynamic full-field strain of an offshore tripod structure during operation with high accuracy. Tarpø et al. [13] proved that the modal expansion technique applies to a subsystem within a nonlinear and time-varying system when the nonlinear and time-varying effects act externally on the subsystem. At the end of the 2010s, machine learning and data-driven models enter the portfolio of stress/strain estimation techniques where Lu et al. [14] applied pattern recognition and Deng et al. [15] applied deep learning to modal expansion.

In this paper, we will study data-driven strain estimation on an operating offshore wind turbine in the Great Belt in Denmark. In general, a data-driven model consists of relationships between input and output of a system and is constructed through training data without any explicit knowledge of the system [16]. Therefore, data-driven strain estimation enables a transformation from physical sensors (input) to strain (output) at locations where temporary strain gauges were placed during the training data. Thus, this form of strain estimation is unextendable to other locations without adding information or new data to the model. This is the main drawback of data-driven strain estimation. Data-driven strain estimation, however, has the advantage over strain gauges in the form of prolonged service life. Strain gauges are known to be unreliable over long-term monitoring - especially in an offshore environment where they are damaged by the corrosive seawater. The service life of the data-driven strain estimation depends on the utilised physical sensors (eg. geophones or accelerometers) and these often have longer service life and are easier to replace than strain gauges. Furthermore, data-driven strain estimation is independent of a system model (often a finite element model) and the accuracy of this model.

In this paper, we utilize training data to construct a data-driven model that can estimate the strain response with high accuracy and reliability. The model works for different wind scenarios than the one from the training data. 


\section{STRAIN ESTIMATION USING PRINCIPAL COMPONENT ANALYSIS}

In this paper, we apply the Principal Component Analysis (PCA) [17] in a supervised learning approach to find a transformation matrix between the displacement (the input) and the strain response of that system (the output) based on training data. Essentially, principal component analysis is a dimensionality reduction of data to set of uncorrelated principal components ordered by contribution [17].

In this technique, we will stack the displacement vector, $\mathbf{y}(t)$, and the strain response, $\boldsymbol{\varepsilon}(t)$, in a vector.

$$
\mathbf{y}_{c}(t)=\left[\begin{array}{l}
\mathbf{y}(t) \\
\varepsilon(t)
\end{array}\right]
$$

We estimate the covariance matrix of this stacked response and apply the singular value decomposition on the covariance matrix.

$$
\mathbf{U S U}^{T}=\mathbb{E}\left[\mathbf{y}_{c}(t) \mathbf{y}_{c}(t)^{T}\right]
$$

where $\mathrm{S}$ is a diagonal matrix holding the singular values, $\mathrm{U}$ holds the singular vectors as column vectors, $\mathbb{E}[\cdot]$ denotes the expectation operator, and $(\cdot)^{T}$ denotes the transpose of a vector or matrix.

We use the singular values, $\mathrm{S}$, to find the dominating principal components to represent the majority of the response. Then we can create a displacement-to-strain matrix, $\mathbf{H}$, using the singular vectors corresponding to these dominating singular components. We can estimate the strain response in other data set by the displacement-to-strain matrix.

$$
\widehat{\varepsilon}(t)=\mathbf{H y}(t)
$$

We must avoid an ill-posed problem, which requires a redundant sensor network with more physical sensors than included singular vectors in $\widehat{\mathbf{U}}$. Fortunately, we can separate the data into multiple frequency bands and find a set of singular vectors for each band.

\section{CASE STUDY}

\subsection{Quality measures}

In the literature or industry, there are no rules or guidelines for an evaluation of virtual sensors. The most common approach is to compare the virtual sensors with a set of reference sensors through different quality measurements. In this paper, we will use two different quality measurements.

\subsubsection{Coefficient of determination $\left(R^{2}\right)$}

The coefficient of determination $\left(R^{2}\right)$ [18] is a popular quality measure in statistics and modal validation where a value of one indicates perfect correlation with the same amplitudes.

$$
R_{i}^{2}=1-\frac{\mathbb{E}\left[\left(\varepsilon_{i}(t)-\widehat{\varepsilon}_{i}(t)\right)^{2}\right]}{\operatorname{Var}\left[\boldsymbol{\varepsilon}_{i}(t)\right]}
$$

where $\varepsilon_{i}(t)$ is the measured strain response for the $i^{t h}$ strain gauge, $\widehat{\varepsilon}_{i}(t)$ is the estimated strain response at the same location, and $\operatorname{Var}[\cdot]$ denotes the variance operator. 


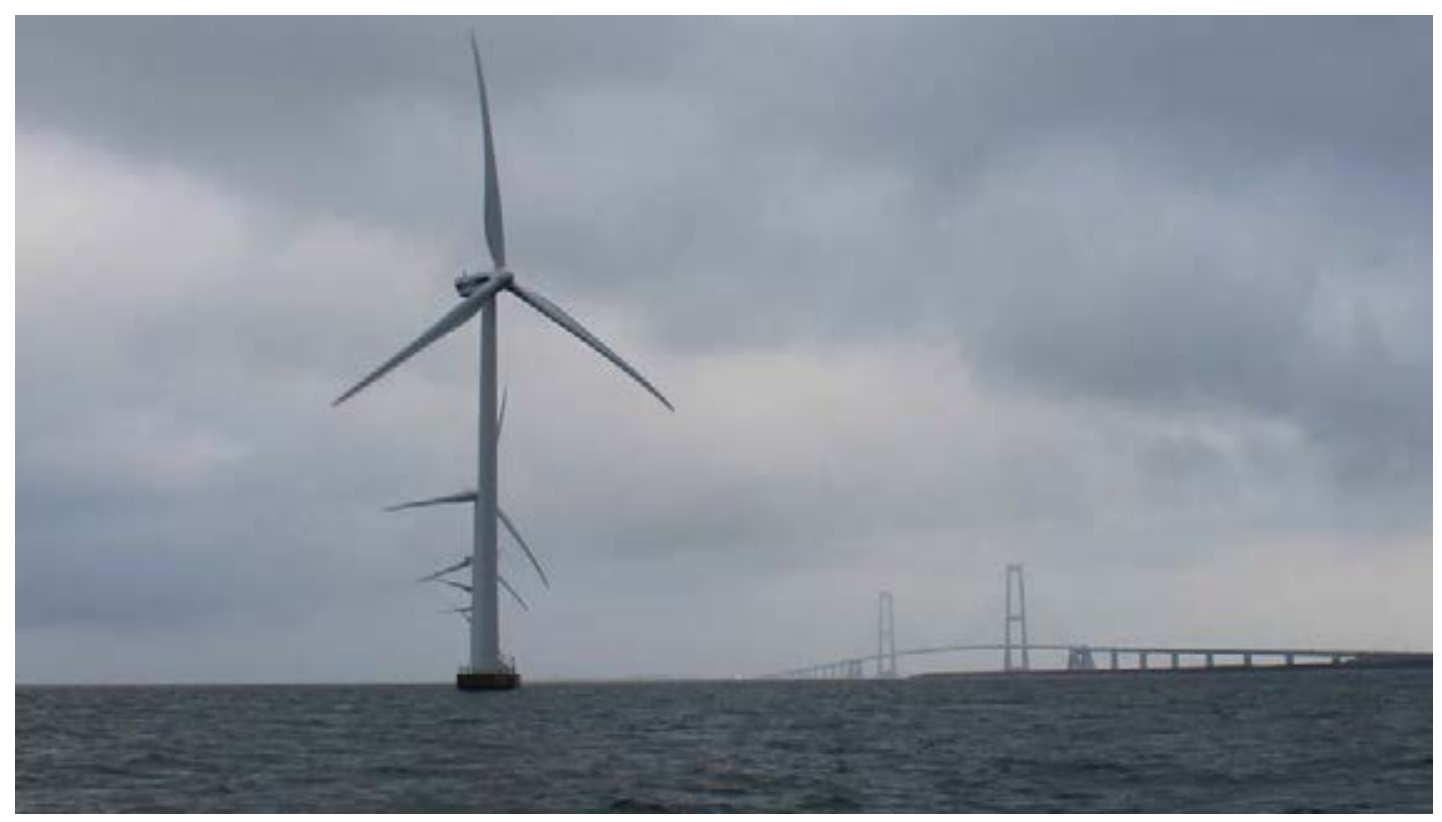

Figure 1: Wind turbine near Sprog $\varnothing$ and the Great Belt Bridge in Denmark

\subsubsection{Normalised error in equivalent stress range}

It is important to evaluate stress estimation in terms of the fatigue damage when the estimation is intended for a fatigue analysis. When we have varying stress amplitudes, we must apply cycle counting [19], like rainflow counting [20]. Sometimes, we might prefer to access fatigue damage for variable amplitude loading in the form of a damage equivalent stress range, which leads to the same fatigue damage as all stress cycles [19]. We calculate the damage equivalent stress range for both reference and the estimated as follows

$$
\Delta \sigma_{e q}=\left(\frac{1}{n_{\text {cycles }}} \sum_{j=1}^{n_{\text {cycles }}} \Delta \sigma_{j}^{m}\right)^{1 / m} \quad, \Delta \hat{\sigma}_{e q}=\left(\frac{1}{\hat{n}_{\text {cycles }}} \sum_{j=1}^{\hat{n}_{\text {cycles }}} \Delta \hat{\sigma}_{j}^{m}\right)^{1 / m}
$$

where $n_{\text {cycles }}$ and $\hat{n}_{\text {cycles }}$ are the total number of counted cycles for the measured and estimated signal, respectively, $\Delta \sigma_{j}$ and $\Delta \hat{\sigma}_{j}$ denote the stress range from cycle counting, and $m$ is the "slope" of the SN curve. For this paper, we will assume $m=3$, which corresponds to welded steel structures [19].

The normalised error of the equivalent stress range is given as

$$
\eta_{i}=\frac{\Delta \hat{\sigma}_{e q}-\Delta \sigma_{e q}}{\Delta \sigma_{e q}}
$$

Here $\eta_{i}=0$ indicates a correct strain estimation in terms of equivalent fatigue damage while a negative value suggests an underestimation and a positive value suggests an overestimation of fatigue damage.

\subsection{Wind turbine}

The case study is an offshore wind turbine in the Great Belt in Denmark and the model of the wind turbine is a Vestas V90, $3 \mathrm{MW}$ installed on a gravitation foundation, see Fig. 1. The wind turbine is equipped with a sensor network consisting of four Sigicom triaxial geophones 

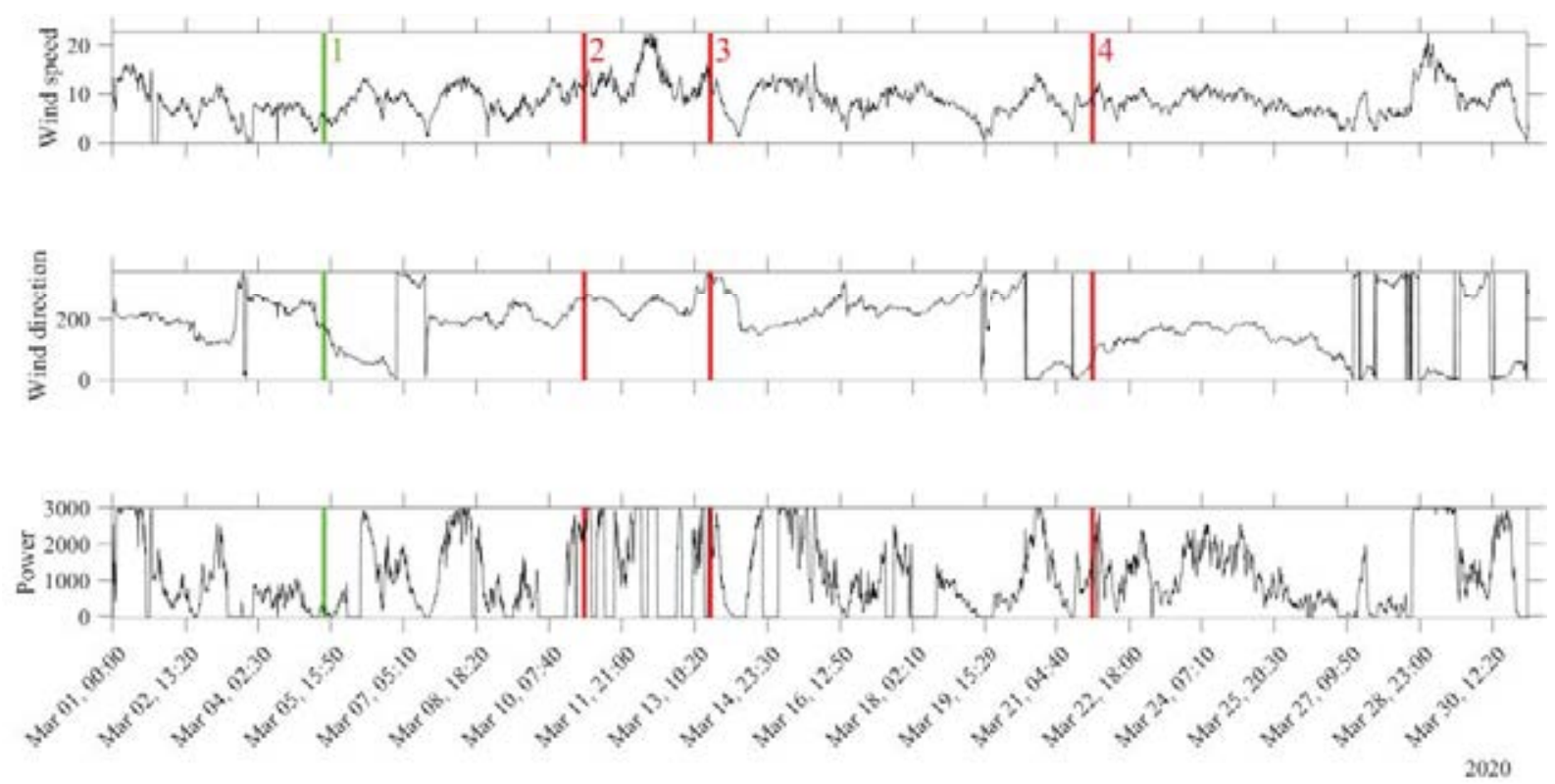

Figure 2: Monitoring campaign during a month with the measured wind speed, wind direction, and the production of power of the wind turbine. The chosen data sets are marked with a line and numbered where the training data set is marked with a green line and the others are marked with red.

attached at the lower part of the tower and two strain gauges located just above the foundation. The monitoring campaign was during a one month period, see Fig. 2, with a sampling frequency of $20 \mathrm{~Hz}$.

To exclude the quasi-static response of the wind turbine, we apply a high-pass filtered to all data with a cut-off frequency of $0.2 \mathrm{~Hz}$. We calibrate the geophones using digital correlation [21] and we reduce the tilt effects on the geophones through the vertical geophones [22]. We integrate signals from the geophones into displacement by the integration theorem for the Fourier transformation [23]. Then, the data is divided into 3 frequency bands: $0.2-0.7,0.7-4.5$, and 4.5-10 Hz using complementary filters. These filters are applied to avoid an ill-posed problem for the strain estimation. We applied 40 minutes of training data to the principal component analysis, as explained in Section 2, to generate the transformation matrix for each frequency band. The training data set is marked with green in Fig. 2 and it has a wind direction of $180^{\circ}$ and a low power production. The results of the strain estimation is shown in Fig. 3 for the training data sets. This displacement-to-strain matrix is saved as the data-driven model for the strain estimation for the entire monitoring period.

To illustrate the accuracy of the data-driven model, we apply the strain estimation model to three other data sets with different wind direction and power production distributed over the entire monitoring campaign, see the data sets marked with red in Fig. 2. Figs. 4, 5, and 6 show the strain estimation in both time and frequency domain for the three data sets. Finally, Fig. 7 (a) shows the coefficient of determination $\left(R^{2}\right)$, Eq. (4), for all three data sets while Fig. 7 (b) displays the normalised error of equivalent strain range, Eq. (6).

All coefficient of determination values are above 0.965 and the estimated equivalent stress range is within $\pm 7 \%$ of the measured one. All these values indicate that the data-driven model estimates the strain response with high accuracy and low uncertainty. Furthermore, strain estimation of the training data has similar quality measurements as the other data sets. This indicates that the displacement-to-strain matrix is versatile and applies to different wind scenarios of wind directions, wind speeds, and power production than the original training data set, 
(a)

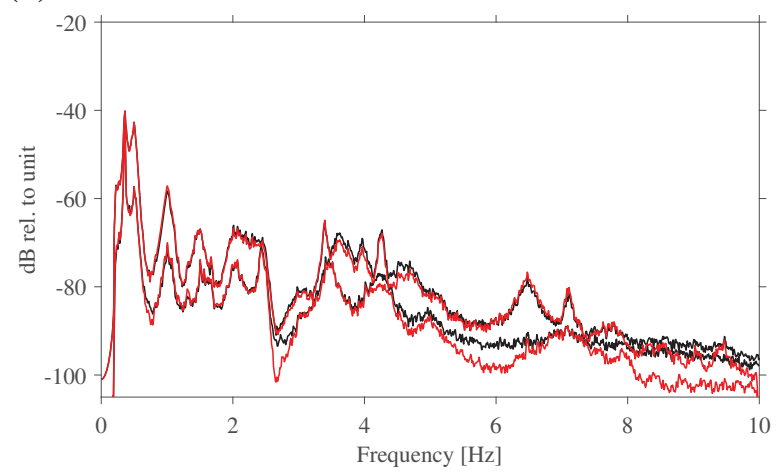

(b)
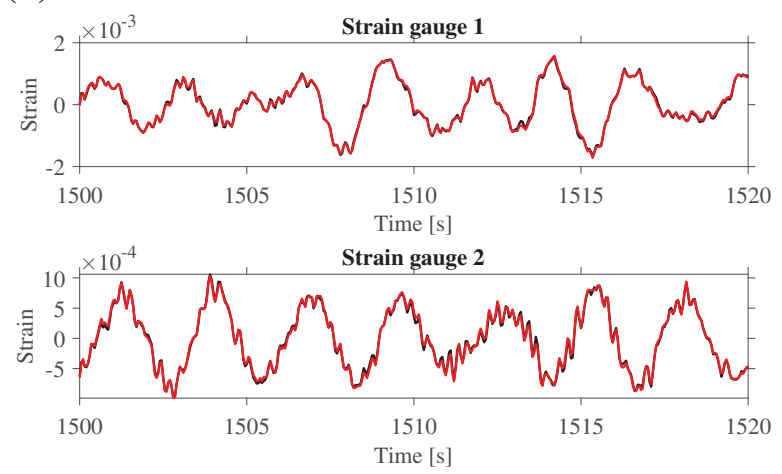

Figure 3: First data-set (training data) corresponding to the marking in Fig. 2 with measured strain (black line) and estimated strain (red line): (a) frequency domain with the first two singular values of the spectral density matrix calculated using Welch averaging method with segments of 2048 data points and $50 \%$ overlap and (b) time domain with a zoom on the strain response.

(a)

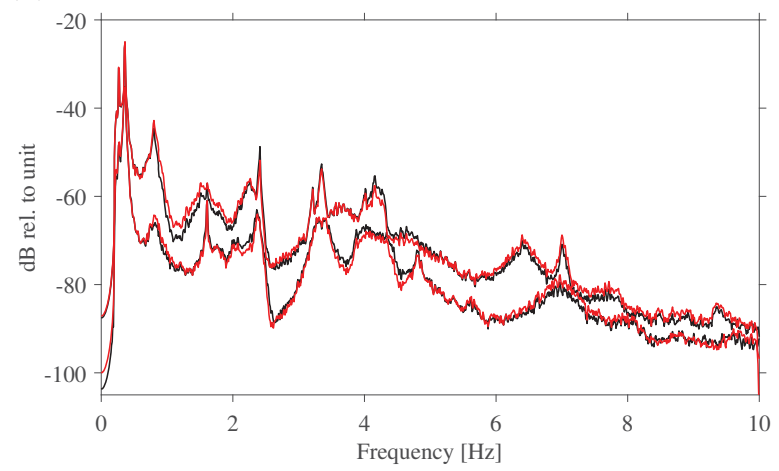

(b)
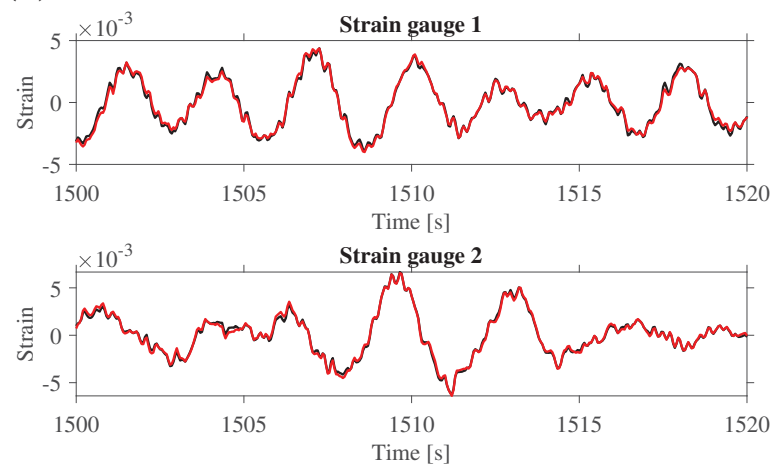

Figure 4: Second data-set corresponding to the marking in Fig. 2 with measured strain (black line) and estimated strain (red line): (a) frequency domain with the first two singular values of the spectral density matrix calculated using Welch averaging method with segments of 2048 data points and $50 \%$ overlap and (b) time domain with a zoom on the strain response.

(a)

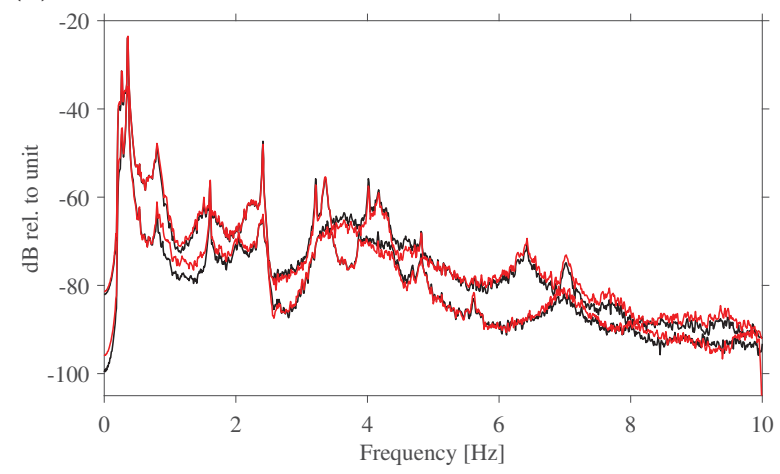

(b)
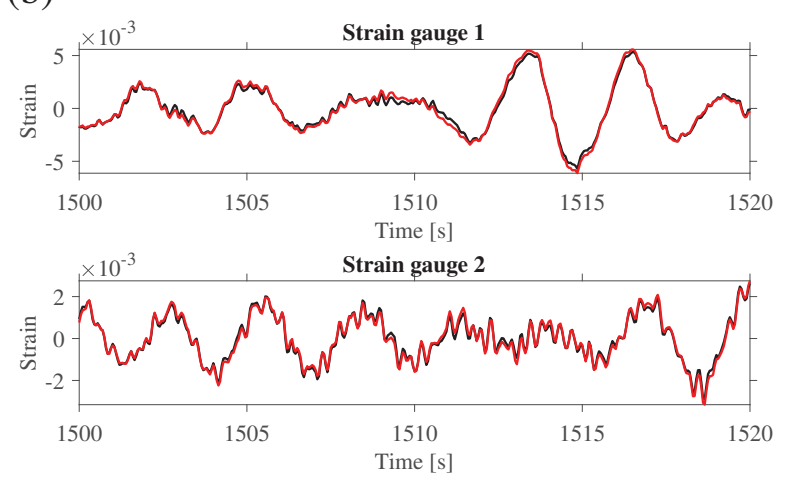

Figure 5: Third data-set corresponding to the marking in Fig. 2 with measured strain (black line) and estimated strain (red line): (a) frequency domain with the first two singular values of the spectral density matrix calculated using Welch averaging method with segments of 2048 data points and $50 \%$ overlap and (b) time domain with a zoom on the strain response. 
(a)

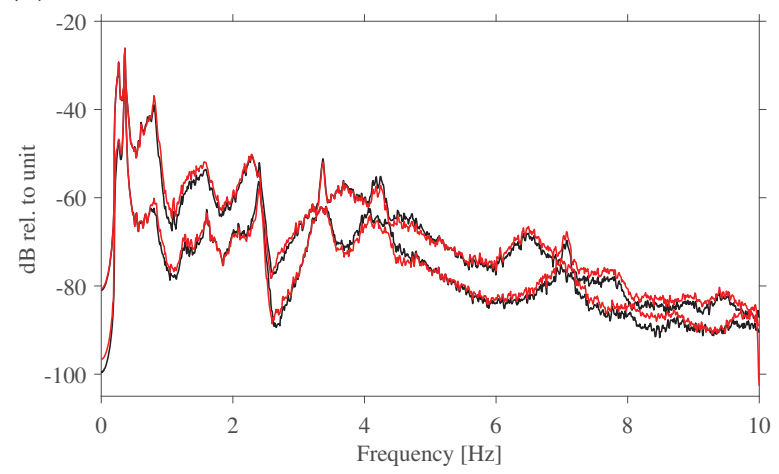

(b)
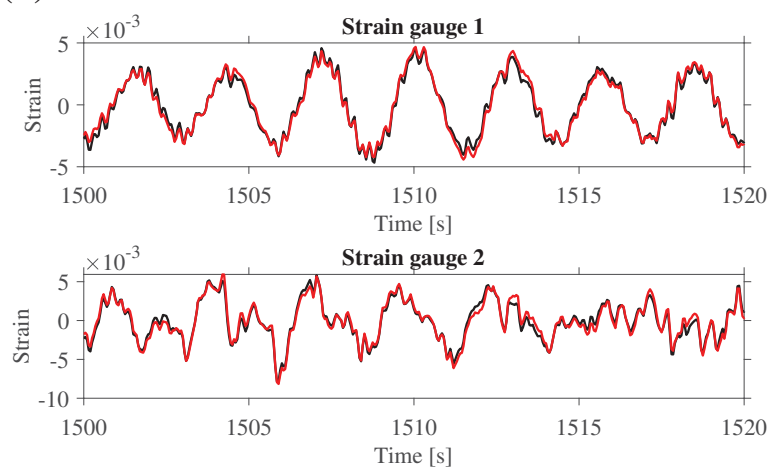

Figure 6: Fourth data-set corresponding to the marking in Fig. 2 with measured strain (black line) and estimated strain (red line): (a) frequency domain with the first two singular values of the spectral density matrix calculated using Welch averaging method with segments of 2048 data points and $50 \%$ overlap and (b) time domain with a zoom on the strain response.

(a)

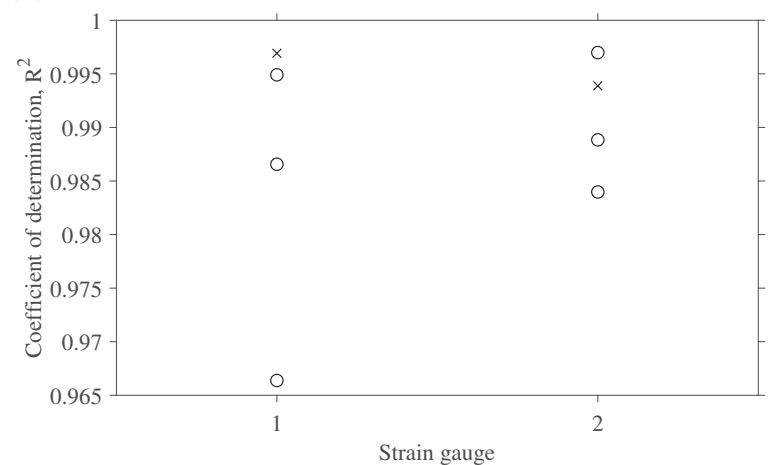

(b)

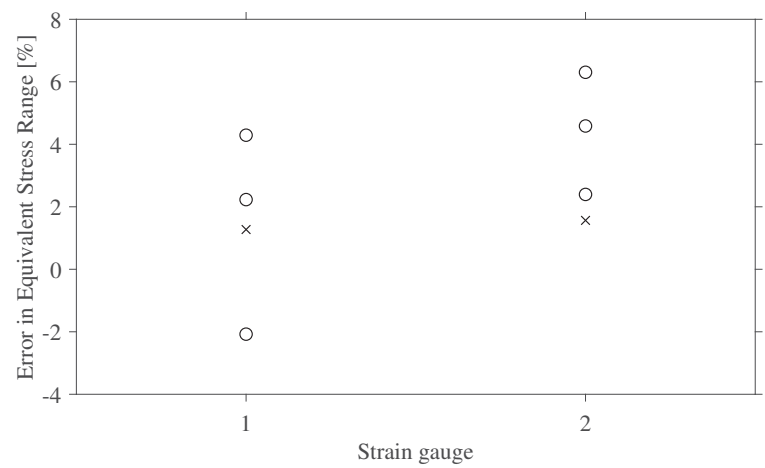

Figure 7: Quality measurements where training data set is marked with $\times$ and the other data sets are marked with $\circ$ : (a) the coefficient of determination $\left(R^{2}\right)$, Eq. (4), for the estimated and measured strain, and (b) the normalised error in equivalent strain range, Eq. (6), for the estimated and measured strain.

which was constructed from.

\section{CONCLUSION}

In this paper, a data-driven strain estimation technique was introduced based on principal component analysis and temporary strain gauges. After the creation of the data-driven model, the temporary strain gauges become unessential for the future monitoring of the strain response and they can be removed. The technique was applied to an offshore wind turbine equipped with both triaxial geophone and strain gauges. A single data set was used to generate the data-driven model that could estimate the strain response - using only vibration responses from the geophones - with high precision in other data sets under different wind scenarios. Recapitulating, the virtual sensors were reliable and robust regardless of wind direction, wind speed, and power production of the wind turbine.

\section{ACKNOWLEDGEMENTS}

The authors acknowledge the funding received from the Centre for Oil and Gas - DTU/Danish Hydrocarbon Research and Technology Centre (DHRTC). 


\section{REFERENCES}

[1] L. Liu, S. M. Kuo, and M. Zhou. Virtual sensing techniques and their applications. In 2009 International Conference on Networking, Sensing and Control, pages 31-36, March 2009.

[2] Marius Tarpø. Stress estimation of offshore structures. PhD thesis, Aarhus University, 2020.

[3] Denis Gordon Karczub. The prediction of dynamic stress and strain in randomly vibrating structures using vibrational velocity measurements. $\mathrm{PhD}$ thesis, The University of Western Australia, 1996. This thesis has been made available in the UWA Profiles and Research Repository as part of a UWA Library project to digitise and make available theses completed before 2003. If you are the author of this thesis and would like it removed from the UWA Profiles and Research Repository, please contact digitaltheses-lib@uwa.edu.au.

[4] N. Okubo and K. Yamaguchi. Prediction of strain distribution under operating condition by the use of modal analysis. Proceedings of the Internation Modal Analysis Conference XIII, 210(5):91-96, 1995.

[5] H.P. Hjelm, R.Brincker, J. Gaugaard-Jensen, and K. Munch. Determination of stress histories in structures by natural input modal analysis. Proceedings of IMAC XXIII Internation Modal Analysis Conference, 12:838-844, 2006.

[6] C. Papadimitriou, C.P. Fritzen, P. Kraemer, and E. Ntotsios. Fatigue predictions in entire body of metallic structures from a limited number of vibration sensors using kalman filtering. Structural Control and Health Monitoring, 18(5):554-573, 2011.

[7] K. Maes, A. Iliopoulos, W. Weijtjens, C. Devriendt, and G. Lombaert. Dynamic strain estimation for fatigue assessment of an offshore monopile wind turbine using filtering and modal expansion algorithms. Mechanical Systems and Signal Processing, 76-77:592-611, 2016.

[8] Alexandros Iliopoulos, Wout Weijtjens, Danny Van Hemelrijck, and Christof Devriendt. Fatigue assessment of offshore wind turbines on monopile foundations using multi-band modal expansion. Wind Energy, 20(8):1463-1479, 2017.

[9] A. Skafte, J. Kristoffersen, J. Vestermark, U. T. Tygesen, and R. Brincker. Experimental study of strain prediction on wave induced structures using modal decomposition and quasi static ritz vectors. Engineering Structures, 136:261-276, 4/1 2017.

[10] Enrico Risaliti, Tommaso Tamarozzi, Martijn Vermaut, Bram Cornelis, and Wim Desmet. Multibody model based estimation of multiple loads and strain field on a vehicle suspension system. Mechanical Systems and Signal Processing, 123:1 - 25, 2019.

[11] M. Tarpø, B. Nabuco, C. Georgakis, and R. Brincker. Expansion of experimental mode shape from operational modal analysis and virtual sensing for fatigue analysis using the modal expansion method. International Journal of Fatigue, 130:105280, 2020.

[12] Bruna Nabuco, Marius Tarpø, Ulf T. Tygesen, and Rune Brincker. Fatigue stress estimation of an offshore jacket structure based on operational modal analysis. Shock and Vibration, 2020:12, 2020. 
[13] Marius Tarpø, Tobias Friis, Christos Georgakis, and Rune Brincker. Full-field strain estimation of subsystems within time-varying and nonlinear systems using modal expansion. Mechanical Systems and Signal Processing, 153:107505, 2021.

[14] Wei Lu, Jun Teng, Qiushi Zhou, and Qiexin Peng. Stress prediction for distributed structural health monitoring using existing measurements and pattern recognition. Sensors, 18(2), 2018.

[15] Huaxia Deng, Haicong Zhang, Jun Wang, Jin Zhang, Mengchao Ma, and Xiang Zhong. Modal learning displacement-strain transformation. Review of Scientific Instruments, 90(7):075113, 2019.

[16] Kevin P. Murphy. Machine Learning: A Probabilistic Perspective. MIT Press, Cambridge, Mass., USA, 2012.

[17] Ian Jolliffe. Principal component analysis. Springer Verlag, New York, USA, 2nd revised edition, 2002.

[18] P. C. Hansen, V. Pereyra, and G. Scherer. Least squares data fitting with applications. Johns Hopkins University Press, Baltimore, MD, 2013.

[19] M.M. Pedersen. Introduction to metal fatigue - concepts and engineering approaches. Technical report, Department of Engineering, Aarhus University, 2018.

[20] C. Amzallag, J.P. Gerey, J.L. Robert, and J. Bahuaud. Standardization of the rainflow counting method for fatigue analysis. International Journal of Fatigue, 16(4):287 - 293, 1994.

[21] Rune Brincker, Thomas Lagö, Palle Andersen, and Carlos Ventura. Improving the classical geophone sensor element by digital correction. 012005.

[22] M. Tarp $\varnothing$, B. Nabuco, R. Boroscek, and R. Brincker. Tilt errors of translational accelerometers attached to dynamic systems with tilt motion caused by the system response. In revision at Journal of Sound and Vibration.

[23] R. Brincker and C. Ventura. Introduction to operational modal analysis. John Wiley and Sons, Inc., Chichester, West Sussex, UK, 2015. 